\title{
An Example of Microstructure with Multiple Scales
}

\author{
Matthias Winter \\ Department of Mathematics \\ Heriot-Watt University \\ Edinburgh, U.K. \\ January 15, 2007
}

\begin{abstract}
This paper studies a vectorial problem in the calculus of variations arising in the theory of martensitic microstructure. The functional has an integral representation where the integrand is a nonconvex function of the gradient with exactly four minima. We prove that the Young measure corresponding to a minimising sequence is homogeneous and unique for certain linear boundary conditions. We also consider the singular perturbation of the problem by higher-order gradients. We study an example of microstructure involving infinite sequential lamination and calculate its energy and length scales in the zero limit of the perturbation.
\end{abstract}

\section{Introduction}

This paper is concerned with the analysis of an example of microstructure which arises by repeating a certain construction step, the so-called lamination again and again. 
Our interest in this problem arises from the modelling of martensitic phase transformations, see e.g. the work by Khachaturyan and Shatalov [9], [10], and Roitburd [17] for a geometrically linear approach or Ball and James [3], [4] for a geometrically nonlinear model. In such transformations fine-scale mixtures of distinct phases or phase variants are possible. The microstructures can be explained by elastic energy minimisation. Minimising the sum of elastic and interfacial energy instead prevents infinite refinement and explains the length scales which are observed in some experiments.

To explain the mathematical issues in more detail consider a variational problem of the form $\left(\boldsymbol{V} \boldsymbol{P}_{0}\right)$ :

minimise

$$
I_{0}(u)=\int_{\Omega} W(\nabla u) d x
$$

among all functions $u \in \mathcal{A}$ where

$$
\mathcal{A}=\left\{u \in W^{1, \infty}(\Omega): u=H x \text { for } x \in \partial \Omega\right\}
$$

$u: \Omega \subset R^{2} \rightarrow R^{2}$ and $\Omega$ is a bounded domain. The integrands $W$ which arise in the study of martensitic phase transformations are nonconvex (and not quasiconvex) in $\nabla u$. Therefore the functional is not lower semicontinuous and the problem typically possesses no minimisers but minimising sequences. A minising sequence $u^{(k)}$ can develop spatial oscillations in its gradients $\nabla u^{(k)}$ which leads to weak rather than strong convergence as $k \rightarrow \infty$. The central idea of energy minimisation is that these oscillations model the microstructures observed in real materials.

We are particularly interested in integrands $W$ which have "multiple well structure", i.e. where $W \geq 0$ and $W=0$ on a known set $\mathcal{K}$. The connected components of $\mathcal{K}$ are the "elastic energy wells". They represent preferred gradients, i.e. stress-free states.

Three different situations can arise. In the first case the minimum of $\left(\boldsymbol{V} \boldsymbol{P}_{\mathbf{0}}\right)$ is achieved and is zero. This occurs, if there is a Lipschitz-continuous deformation $u(x)$ satisfying the boundary condition, such that $\nabla u(x) \in \mathcal{K}$ for almost every $x$. Such a deformation is called stress-free. 
Second it can happen that the infimum of $\left(\boldsymbol{V} \boldsymbol{P}_{\mathbf{0}}\right)$ might be 0 but is not achieved. Then a minising sequence $u^{(k)}$ has the property that $\nabla u^{(k)}$ is approximately in $\mathcal{K}$ except in a subset of the domain whose measure tends to zero as $k \rightarrow \infty$. (This will be formalised using the notion of a Young measure.) We can think of $\nabla u^{(k)}$ as determining a microstructure with a length scale that gets finer as $k \rightarrow \infty$. When $k$ is large $\nabla u^{(k)}$ subdivides the domain into regions which are nearly stress-free, i.e. where $\nabla u^{(k)}$ is near $\mathcal{K}$ and and in "transition layers" where $\nabla u^{(k)}$ is not near $\mathcal{K}$ but bounded independent of $k$ and which are of small measure. Such a microstructure is called essentially stress-free.

In the third situation the infimum of $\left(\boldsymbol{V} \boldsymbol{P}_{\mathbf{0}}\right)$ is not 0 . In this situation there may or may not be a deformation $u(x)$ for which the minimum is achieved. Such a system is typically stressed.

Since we are interested in weakly convergent sequences, it is convenient to use the notion of a Young measure, which is mainly an accounting device. Intuitively, the Young measure $\nu_{x}$ associated to an oscillatory sequence is a family of probability measures parametrised with respect to the points $x \in \Omega$ which gives the limiting probability distribution of the values of the gradient of $u$ near $x$, in the limit as $k \rightarrow \infty$. (A more formal definition is given in Lemma 2.1 below.)

The study of Young measures is linked to other fundamental mathematical questions. A basic problem in the calculus of variations is to find necessary and sufficient conditions for quasiconvexity. Kinderlehrer and Pedregal have shown that this is linked to determining the set of possible Young measure limits of gradients [11].

A very important subclass of gradient Young measures consists of the laminates. Most of the examples observed in experiments are of this type. We are not going to give a formal and most general definition of a laminate (which can be found for example in the paper of Pedregal [16]). Instead, we first define what we mean by a simple laminate and then introduce an (infinitely) sequentially laminated microstructure by recursion.

If we have a deformation $u: \Omega \subset R^{2} \rightarrow R^{2}$ whose gradient $\nabla u$ takes on the given values $A, B \in M^{2 \times 2}\left(M^{2 \times 2}\right.$ is the set of all $2 \times 2$ matrices $)$. on either side of a planar interface then 
we need the two phases to be kinematically compatible, i.e. the difference matrix should be of rank 1 or $A-B=a \otimes n$ where $a \otimes n$ is the matrix $(a \otimes n)_{i j}=a_{i} n_{j}$ for $a, n \in R^{2}$ and $n$ is the normal to the interface (see Ball and James [3]). Physically, this rank-one compatibility gives continuity of $u$ across the interface. For some $\lambda \in(0,1)$ set $H=\lambda A+(1-\lambda) B$. Following Chipot and Kinderlehrer [7] let $\chi$ be the characteristic function of $(0, \lambda)$ in $(0,1)$ extended periodically to the real line $R$ and write

$$
f^{(k)}(x)=\chi(k n \cdot x), \quad k=1,2, \ldots
$$

It is well-known that $f^{(k)} \stackrel{*}{\rightarrow} \lambda$ in $L^{\infty}(\Omega)$ for any bounded $\Omega \subset R^{2}$. Let

$$
u(\xi)=B \xi+\int_{0}^{\xi \cdot n} \chi(t) d t \cdot a
$$

and

$$
u^{(k)}(x)=\frac{1}{k} u(k x)
$$

Then

$$
\nabla u^{(k)}=\left(1-f^{(k)}\right) A+f^{(k)} B .
$$

Note that the gradient $\nabla u^{(k)}$ takes the values $A$ and $B$ in alternate layers of thickness $\lambda / k$ and $(1-\lambda) / k$, i.e. the layer width tends to zero as $k \rightarrow \infty$. Then $\nu=(1-\lambda) \delta_{A}+\lambda \delta_{B}$ is the Young measure corresponding to the sequence $\left\{\nabla u^{(k)}\right\}$, i.e. it satisfies

$$
\lim _{k \rightarrow \infty} \int_{E} \varphi\left(\nabla u^{(k)}\right) d x+|E|((1-\lambda) \varphi(A)+\lambda \varphi(B))=\int_{E} \int_{M^{2 \times 2}} \varphi(Y) d \nu_{x}(Y) d x
$$

for any continuous $\varphi$ and measurable $E \subset \Omega$. Throughout the paper $\delta_{Y}$ stands for the Dirac mass at $Y \in M^{2 \times 2}$.

Assume now that $B=\theta C+(1-\theta) D$ with $C-D$ of rank one. Then we apply the process of singe lamination again thus replacing the gradients $B$ by gradients $C$ and $D$ with volume proportions $\theta$ and $1-\theta$. The resulting Young measure is

$$
\nu=\lambda \delta_{A}+(1-\lambda) \theta \delta_{C}+(1-\lambda)(1-\theta) \delta_{D} .
$$

We have to do something slightly different in order to obtain a continuous deformation. But these technical details can be taken care of and they do not affect the Young measure. 
We define an (infinitely) sequentially-laminated microstructure as one which can be obtained by starting from a compact set $\mathcal{K}$ of matrices and repeating the lamination step (infinitely) many times.

In this paper we revisit an example introduced independently by Aumann and Hart [1] and Tartar [20]. It is an interesting example of the second situation mentioned above, i.e. of an essentially stress-free microstructure. (This fact was proved in [8], Proposition 2.2.)

In [5] Bhattacharya, Firoozye, James, and Kohn explicitly give a minimising sequence based on infinite sequential lamination and calculate its Young measure.

Now we introduce the mathematical framework for this study.

Conditions: $1 . W(X) \geq 0$ for all $2 \times 2$ matrices $X$.

2. There exist four $2 \times 2$ matrices $A, B, C$ and $D$ such that

$$
W(A)=W(B)=W(C)=W(D)=0
$$

and $W>0$ elsewhere.

3. $W$ is continuous.

4. There exist $c, C>0$ and $d, D$ real such that

$$
c|A|^{2}+d \leq W(A) \leq C|A|^{2}+D
$$

where $|A|^{2}=\sum_{\iota, \kappa=1}^{2} a_{\iota \kappa}^{2}$.

5. The matrices $A, B, C$, and $D$ are incompatible, i.e. the difference between any pair of them has rank two.

6. There exist matrices $J_{1}, J_{2}, J_{3}$, and $J_{4}$, vectors $a, b, c, d \in R^{m}$, vectors $p, q, r, s \in R^{n}$ and scalars $\alpha, \beta, \gamma, \delta \in(0,1)$ such that

$$
\begin{array}{ll}
J_{1}=\alpha A+(1-\alpha) J_{2}, & J_{2}=\beta B+(1-\beta) J_{3}, \\
J_{3}=\gamma C+(1-\gamma) J_{4}, & J_{4}=\delta D+(1-\delta) J_{1} ;
\end{array}
$$




$$
\begin{array}{ll}
A-J_{2}=a \otimes p, & B-J_{3}=b \otimes q, \\
C-J_{4}=c \otimes r, & D-J_{1}=d \otimes s ;
\end{array}
$$

see Figure 1.

We present two new results. First we prove a theorem about the Young measure of the gradients of any minimising sequence.

It is clear from the definition of the Young measure that it has to be supported on the set $\{A, B, C, D\}$. We further assume certain linear boundary conditions and show that the Young measure is homogeneous and unique and satisfies

$$
\nu_{x}=\lambda_{1} \delta_{A}+\lambda_{2} \delta_{B}+\lambda_{3} \delta_{C}+\lambda_{4} \delta_{D} \quad \text { a.e. in } \Omega \text {. }
$$

It is easy to see that the Young measure corresponding to the infinitely sequentially-laminated construction is homogeneous. The theorem proves with mathematical rigour that this feature must be shared by any minimising sequence.

Second we consider the following variational problem which arises from $\left(\boldsymbol{V} \boldsymbol{P}_{\mathbf{0}}\right)$ by singular perturbation with higher-order gradients and is denoted by $\left(\boldsymbol{V} \boldsymbol{P}_{\boldsymbol{\varepsilon}}\right)$ : minimise

$$
I_{\varepsilon}(u)=\int_{\Omega} W(\nabla u)+\varepsilon^{2}|\nabla \nabla u|^{2} d x
$$

among all functions $u \in \mathcal{A}_{K}$ where

$$
\begin{gathered}
\mathcal{A}_{K}=\left\{u \in H^{2}(\Omega): \nabla u \in L^{\infty}(\Omega),\|\nabla u\|_{\infty} \leq K,\right. \\
u=H x \text { on } \partial \Omega\},
\end{gathered}
$$

$K \geq 3,|\nabla \nabla u|^{2}=\sum_{\iota, \kappa, \xi=1}^{2}\left(\partial_{\iota} \partial_{\kappa} u_{\xi}\right)^{2}$, and $u: \Omega \subset R^{2} \rightarrow R^{2}$. This problem is physically interesting since the perturbation terms correspond to surface energy. The inclusion of surface energy terms into the problem introduces a length scale and, furthermore, because of convexity and coercivity in the highest derivatives the problem now has a minimiser. Since surface energy is typically very small it is physically meaningful to study the behaviour of the energy minimum in the limit of zero surface energy. 
We study this question by introducing length scales into the infinitely sequentially-laminated microstructure considered in [5].

Now we give a simplified presentation of the main ideas. The argument is based on appropriate scaling. For a (simply-)laminated microstructure on a two-dimensional domain the energy behaves like

$$
E_{\varepsilon} \sim \varepsilon^{1-\alpha}+\varepsilon^{\alpha} \quad \text { as } \varepsilon \rightarrow 0
$$

if the distance between two interfaces is of the order $\varepsilon^{\alpha}$. (The symbol $a \sim b$ is a shorthand for the following: There exist constants $c, C>0$ such that $c b \leq a \leq C b$.) The first term in the energy represents surface energy of the interfaces, the second refinement near the domain boundary. Choosing $\alpha=1 / 2$ we get

$$
E_{\varepsilon} \sim \varepsilon^{1 / 2}
$$

In [12], [13], [14] Kohn and Müller show that by choosing instead a two-dimensional construction with refinement near the domain boundary the asymptotic behaviour $E_{\varepsilon} \sim \varepsilon^{2 / 3}$ can be achieved and that the exponent is optimal. In [21] their model is revisited and a Lavrentiev phenomenon is proved.

The present situation is more complex since simple lamination is not enough to achieve gradients with small energy density $W$ except at the interface regions and near the domain boundary. Instead we now have to consider a construction which uses sequential lamination and produces multiple scales. The corresponding energy is

$$
E_{\varepsilon} \sim \frac{1}{2^{k}}+\sum_{i=1}^{k-1} \frac{1}{2^{i}}\left(\varepsilon^{\alpha_{i+1}-\alpha_{i}}\right)
$$

if we consider $k$ different scales and choose the $i$ th scale $l_{i}$ such that

$$
l_{i} \sim \varepsilon^{\alpha_{i}}
$$

To minimise $E_{\varepsilon}$ in $k, \alpha_{0}, \ldots, \alpha_{k}$ for fixed $\varepsilon$ we first balance the terms in the sum which results in

$$
\alpha_{i}=i \mu-\frac{(i-1) i}{2} \tau
$$


where

$$
\tau \approx \frac{\log 2}{\log (1 / \varepsilon)}
$$

(Note that then

$$
\left.\frac{1}{2^{i}}\left(\varepsilon^{\alpha_{i+1}-\alpha_{i}}\right) \approx \varepsilon^{\mu} .\right)
$$

Second we balance the first term and the terms in the sum, i.e. we choose $k$ and $\mu$ such that

$$
\frac{1}{2^{k}} \approx \varepsilon^{\mu}
$$

and choose the smallest length scale $l_{k}$ to be of the thickness $\varepsilon$ of the interfaces, i.e.

$$
\alpha_{k} \approx 1
$$

The last two conditions can be rewritten as

$$
\mu=k \frac{\log 2}{\log (1 / \varepsilon)}
$$

and

$$
\frac{k^{2}}{2} \frac{\log 2}{\log (1 / \varepsilon)} \approx 1
$$

This implies

$$
k \approx \sqrt{\frac{2}{\log 2}} \sqrt{\log (1 / \varepsilon)}
$$

and

$$
\mu \approx \sqrt{2 \log 2} \frac{1}{\sqrt{\log (1 / \varepsilon)}}
$$

Then

$$
\frac{1}{2^{k}} \approx \exp (-\sqrt{2 \log 2} \sqrt{\log (1 / \varepsilon)})
$$

and

$$
\varepsilon^{\mu} \approx \exp (-\sqrt{2 \log 2} \sqrt{\log (1 / \varepsilon)}) .
$$

But the number of these terms increases to $\infty$ as $\varepsilon$ approaches zero.

An exact analysis reveals that in fact

$$
E_{\varepsilon} \leq C \exp (-\tau \sqrt{\log (1 / \varepsilon)}) \quad \text { for some } C>0 \text { and } 0<\tau<\sqrt{2 \log 2}
$$


and that the constant $\sqrt{2 \log 2}$ is optimal. Note that the decay is slower than any power of $\varepsilon$ (which is the case for simple laminates) but faster than (any power of) the logarithm of $1 / \varepsilon$.

To make the presentation transparent but without loss of generality we choose the following values for the eight matrices:

$$
A=\left(\begin{array}{ll}
1 & 0 \\
0 & 3
\end{array}\right), B=\left(\begin{array}{cc}
3 & 0 \\
0 & -1
\end{array}\right), C=\left(\begin{array}{rr}
-1 & 0 \\
0 & -3
\end{array}\right), D=\left(\begin{array}{rr}
-3 & 0 \\
0 & 1
\end{array}\right),
$$

and

$$
J_{1}=\left(\begin{array}{cc}
1 & 0 \\
0 & 1
\end{array}\right), J_{2}=\left(\begin{array}{rr}
1 & 0 \\
0 & -1
\end{array}\right), J_{3}=\left(\begin{array}{rr}
-1 & 0 \\
0 & -1
\end{array}\right), J_{4}=\left(\begin{array}{rr}
-1 & 0 \\
0 & 1
\end{array}\right)
$$

In particular, all eight matrices are chosen to be $2 \times 2$ and diagonal. As boundary condition we consider

$$
u=J_{1} x \quad \text { on } \partial \Omega \text {. }
$$

This choice does not restrict generality, either. In fact, it is easy to see that the main results of this paper, namely Theorems 2.1 and 3.1 hold for the boundary condition

$$
u=H x \quad \text { on } \partial \Omega
$$

where $H$ is any matrix on the boundary of the diagram given in Figure 1 . Under the assumptions (1.6) and (1.7) (or, more generally, if the matrices $A, B, C, D, J_{1}, J_{2}, J_{3}, J_{4}$ are $2 \times 2$ matrices and diagonal) $H$ can even lie inside or on the boundary of the diagram given in Figure 1 (except for $A, B, C, D$ of course). Furthermore, choose

$$
\Omega=R_{L, H}=(0, L) \times(0, H) .
$$

Our results still hold for any smooth domain.

Note that the smallest number of incompatible matrices which can support a nontrivial Young measure is four, as was proved in [5]. This is one of the reasons for choosing this 
example. This minimality property should be closely connected to the uniqueness of the Young measure.

It was suggested in [5] to interpret the minimal energy of the perturbed problem as a quantitative "measure" for the complexity of the corresponding microstructure, which for our infinitely sequentially-laminated microstructure is

$$
C \exp (-\sigma \sqrt{\log (1 / \varepsilon)})
$$

where $C>0$ and $0<\sigma<\sqrt{2 \log 2}$ are both independent of $\varepsilon$. (At least this expression is an upper bound for the minimal energy). We expect that such a result holds in general, i.e. that the energy of any infinitely sequentially-laminated microstructure behaves like

$$
C \exp (-D \sqrt{\log (1 / \varepsilon)})
$$

with positive real constants $C$ and $D$, even if the microstructure does not arise from the variational problem studied here, but from any singularly perturbed variational problem of the form (1.5).

In a related paper [6] Cellina and Perrotta study the two-well problem. More precisely, they assume that $W$ is zero on two three-dimensional wells $S O(3) A$ and $S O(3) B$ where $A$ is positive definite symmetric and $B$ is indefinite and symmetric. They assume zero boundary conditions. An explicit construction is given using refinement near the boundary of a Lipschitz function whose gradient is in the zero set of $W$ for a.e. $x \in \Omega$.

After the present paper was finished we learned about the work of Chipot [8]. It contains numerical simulations for the unperturbed problem, which show an infinitely sequentiallylaminated microstructure similar to the one considered in Section 3. Furthermore, a uniqueness result for Young measures is obtained, which is similar to ours.

The structure of the paper is as follows. In Section 2 we prove the theorem about Young measures. In Section 3 we provide an upper bound for the asymptotic behaviour of the energy minimum in the zero limit of surface energy. We also calculate the length scales of the corresponding infinitely sequentially-laminated microstructure. 


\section{Uniqueness of the Young measure}

In this section we study the Young measure corresponding a minimising sequence of the variational problem $\left(\boldsymbol{V} \boldsymbol{P}_{\mathbf{0}}\right)$ :

minimise

$$
I_{0}(u)=\int_{\Omega} W(\nabla u) d x
$$

among all functions $u \in \mathcal{A}$ where

$$
\mathcal{A}=\left\{u \in W^{1, \infty}\left(R_{L, H}\right): u=J_{1} x \text { for } x \in \partial \Omega\right\}
$$

and $W$ satisfies (1.2) and (1.3). In particular, we prove that this Young measure is homogeneous and unique. First recall the existence theorem for Young measures.

Lemma 2.1 Suppose that $F^{(k)} \subset L^{\infty}\left(\Omega, R^{s}\right)$ is a sequence of vector-valued functions on $\Omega$. Assume further that for some compact set $\mathcal{K} \subset R^{s}$, the values of $F^{(k)}$ are "asymptotically in $\mathcal{K}$ " in the sense that for every open $U \supset \mathcal{K}$, meas $\left\{x \in \Omega: F^{(k)} \notin U\right\} \rightarrow 0$ as $k \rightarrow \infty$. Then there exists a subsequence (still denoted by $F^{(k)}$ for convenience) and an associated family of probability measures $\nu_{x}$ on $R^{s}$ (parametrised by $x \in \Omega$ ), such that

i) $\nu_{x}$ is supported on $\mathcal{K}$ for a.e. $x$, and

ii) for any continuous function $\Psi$ defined on $R^{s}, \Psi\left(F^{(k)}\right)$ converges to the function $x \mapsto$ $\int_{R^{s}} \Psi(A) d \nu_{x}(A)$ weak ${ }^{*}$ in $L^{\infty}(\Omega)$.

See for example Ball [2] or Tartar [19] for proofs and discussion. In the variational problem ( $\left.\boldsymbol{V} \boldsymbol{P}_{\mathbf{0}}\right)$ the sequence $\left\{F^{(k)}\right\}$ has the form $F^{(k)}=\nabla u^{(k)}$ where $u^{(k)}$ is a minimising sequence. Therefore the corresponding Young measure is called a gradient Young measure. Note that the energy minimum of $\left(\boldsymbol{V} \boldsymbol{P}_{\mathbf{0}}\right)$ is

$$
\lim _{k \rightarrow \infty} \int_{\Omega} W\left(\nabla u^{(k)}(x)\right) d x=\int_{\Omega} \int_{\mathcal{K}} W(A) d \nu_{x}(A) d x
$$

In particular, if $W \geq 0$ and $W(F)=0$ exactly for $F \in \mathcal{K}$, then $\int_{\Omega} W\left(\nabla u^{(k)}(x)\right) d x \rightarrow 0$ if and only if $\nu_{x}$ is supported on $\mathcal{K}$ for a.e. $x \in \Omega$. The main result of this section is the following: 
Theorem 2.1 Let $\nu_{x}$ be a gradient Young measure which

i) arises from a sequence $u^{(k)}$ defined on $R_{L, H}$ satisfying the boundary condition $u^{(k)}=J_{1} x$ at $\partial R_{L, H}$ and

ii) is supported on the four matrices $A, B, C, D$ as defined in (1.6).

Then

$$
\nu_{x}=\lambda_{1} \delta_{A}+\lambda_{2} \delta_{B}+\lambda_{3} \delta_{C}+\lambda_{4} \delta_{D} \quad \text { a.e. in } R_{L, H} .
$$

Furthermore, the weights $\lambda_{1}, \lambda_{2}, \lambda_{3}, \lambda_{4}$ are unique.

Remark: The statement of Theorem 2.1 clearly implies that the Young measure is unique and homogeneous.

Proof. Consider the function $z \in W^{1, \infty}\left(\Omega, R^{2}\right)$ which satisfies

$$
\nabla z(x)=\int_{M^{2 \times 2}} A d \nu_{x}(A) \quad \text { a.e. in } R_{L, H} .
$$

(The existence of the function $z$ was proved by Kinderlehrer and Pedregal [11]). Because $\nu_{x}$ is by assumption supported on $\{A, B, C, D\}$ we have

$$
\nu_{x}=\lambda_{1 x} \delta_{A}+\lambda_{2 x} \delta_{B}+\lambda_{3 x} \delta_{C}+\lambda_{4 x} \delta_{D} \quad \text { a.e. in } R_{L, H} .
$$

In the sequel we determine the scalar measures $\lambda_{i x}$ for $i=1,2,3,4$. Because $\nu_{x}$ is a probability measure we have

$$
\lambda_{1 x}+\lambda_{2 x}+\lambda_{3 x}+\lambda_{4 x}=1 \quad \text { a.e. in } R_{L, H} .
$$

Using the specific values of the matrices $A, B, C, D$ as defined in (1.6) we get

$$
\nabla z(x)=\left(\begin{array}{cc}
\lambda_{1 x}+3 \lambda_{2 x}-\lambda_{3 x}-3 \lambda_{4 x} & 0 \\
0 & 3 \lambda_{1 x}-\lambda_{2 x}-3 \lambda_{3 x}+\lambda_{4 x}
\end{array}\right)
$$

a.e. in $R_{L, H}$. This implies $\frac{\partial z_{1}}{\partial x_{2}}=\frac{\partial z_{2}}{\partial x_{1}}=0$ a.e. in $R_{L, H}$. By standard trace theorems

$$
z(x)=J_{1} x \quad \text { a.e. on } \partial R_{L, H} .
$$


Therefore

$$
\begin{array}{lll}
\left.\frac{\partial z_{1}}{\partial x_{1}}\right|_{x_{2}=0}=\left.\frac{\partial z_{1}}{\partial x_{1}}\right|_{x_{2}=s}=1 & \text { for } 0 \leq s \leq H, \text { a.e. on }(0, L), \\
\left.\frac{\partial z_{2}}{\partial x_{2}}\right|_{x_{1}=0}=\left.\frac{\partial z_{2}}{\partial x_{2}}\right|_{x_{1}=t}=1 & \text { for } 0 \leq t \leq L, \text { a.e. on }(0, H) .
\end{array}
$$

It follows that

$$
\begin{array}{ll}
\lambda_{1 x}+3 \lambda_{2 x}-\lambda_{3 x}-3 \lambda_{4 x}=1 & \text { a.e. in } R_{L, H}, \\
3 \lambda_{1 x}-\lambda_{2 x}-3 \lambda_{3 x}+\lambda_{4 x}=1 & \text { a.e. in } R_{L, H} .
\end{array}
$$

Finally we get a fourth equation for $\lambda_{i x}, i=1,2,3,4$, the "minors relation". This is a consequence of the fact that $\nu_{x}$ is a gradient Young measure, see for example [5]. It is

$$
\begin{aligned}
& \lambda_{1 x} \operatorname{det} A+\lambda_{2 x} \operatorname{det} B+\lambda_{3 x} \operatorname{det} C+\lambda_{4 x} \operatorname{det} D= \\
& \quad=\operatorname{det}\left(\lambda_{1 x} A+\lambda_{2 x} B+\lambda_{3 x} C+\lambda_{4 x} D\right) \quad \text { a.e. in } R_{L, H} .
\end{aligned}
$$

Equations (2.2)-(2.4) are equivalent to the following three equations:

$$
\begin{array}{lr}
\lambda_{2 x}=-\lambda_{1 x}+0.8 & \text { a.e. in } R_{L, H}, \\
\lambda_{3 x}=\lambda_{1 x}-0.4 & \text { a.e. in } R_{L, H}, \\
\lambda_{4 x}=-\lambda_{1 x}+0.6 & \text { a.e. in } R_{L, H} .
\end{array}
$$

Inserting (2.6)-(2.8) into (2.5) gives the linear equation

$$
12 \lambda_{1 x}-6.4=0 \text { a.e. }
$$

The linear system (2.6)-(2.9) has a unique solution. Therefore the weights $\left(\lambda_{1}, \lambda_{2}, \lambda_{3}, \lambda_{4}\right)$ are independent of $x$ and unique, i.e. the Young measure $\nu$ is homogeneous and unique. This concludes the proof of Theorem 2.1.

Note that the result of Theorem 2.1 holds if we take any matrices $A, B, C$, and $D$ which satisfy the equations given in condition 6 of the introduction even if they do not take on the specific values chosen in (1.6). 


\section{Energy minimum and length scales for the microstruc- ture}

In this section we calculate an upper bound for the scaling law of the energy minimum of the variational problem $\left(\boldsymbol{V} \boldsymbol{P}_{\boldsymbol{\varepsilon}}\right)$ as $\varepsilon \rightarrow 0$. This will be accomplished by incorporating length scales into an infinitely sequentially-laminated microstructure. Recall the variational problem $\left(\boldsymbol{V} \boldsymbol{P}_{\varepsilon}\right)$ :

minimise

$$
I_{\varepsilon}(u)=\int_{\Omega} W(\nabla u)+\varepsilon^{2}|\nabla \nabla u|^{2} d x
$$

among all functions $u \in \mathcal{A}_{K}$ where

$$
\begin{gathered}
\mathcal{A}_{K}=\left\{u \in H^{2}\left(R_{L, H}\right): \nabla u \in L^{\infty}\left(R_{L, H}\right),\|\nabla u\|_{\infty} \leq K,\right. \\
\left.u=J_{1} x \text { on } \partial R_{L, H}\right\}
\end{gathered}
$$

and $K \geq 3$.

As the class of admissible functions we choose

$$
\begin{gathered}
\mathcal{A}_{K}=\left\{u \in H^{2}\left(R_{L, H}\right): \nabla u \in L^{\infty}\left(R_{L, H}\right),\|\nabla u\|_{\infty} \leq K,\right. \\
\left.u=J_{1} x \text { on } \partial R_{L, H}\right\}
\end{gathered}
$$

where $K \geq 3$.

Proposition 3.1 For $\varepsilon>0, K \geq 3$ the variational problem

$$
\min \left\{I_{\varepsilon}(u): u \in \mathcal{A}_{K}\right\}
$$

attains its minimum.

Proof: The proof is a standard one in the direct calculus of variations.

Let us now announce the main result of this section. 
Theorem 3.1 For every constant $\sigma$ with $0<\sigma<\sqrt{2 \log 2}$ there exist constants $C>0$ and $\varepsilon_{0}>0$ such that for all $0<\varepsilon<\varepsilon_{0}$ and $K \geq 3$

$$
\min \left\{I_{\varepsilon}(u): u \in \mathcal{A}_{K}\right\} \leq C \exp \left(-\sigma \sqrt{\log \frac{1}{\varepsilon}}\right) .
$$

Proof. We construct test functions $u \in \mathcal{A}_{K}$ depending on $\varepsilon$ such that for them the estimate given in (3.2) holds true. To this end, we introduce a "sharp" variational problem which is closely related to the "diffusional" one given in $\left(\boldsymbol{V} \boldsymbol{P}_{\boldsymbol{\varepsilon}}\right)$ and is denoted by $\left(\widetilde{\boldsymbol{V} \boldsymbol{P}_{\boldsymbol{\varepsilon}}}\right)$ : minimise

$$
E_{\varepsilon}(v)=\int_{R_{L, H}} W(\nabla v)+\varepsilon|\nabla \nabla v| d x
$$

among all functions $v \in \mathcal{B}_{K}$ where

$$
\mathcal{B}_{K}=\left\{v \in H^{1}\left(R_{L, H}\right): \nabla v \in L^{\infty}\left(R_{L, H}\right),\|\nabla v\|_{\infty} \leq K\right.
$$

the components of $\nabla \nabla v$ are Radon measures on $R_{L, H}$ with finite mass,

$$
\left.v=J_{1} x \text { on } \partial R_{L, H}\right\}
$$

The latter problem has advantages over the former: we can prove a result corresponding to (3.2) by constructing $\varepsilon$-dependent test functions $v$ that are piecewise linear and can be defined explicitly.

Again we have existence of a minimiser:

Proposition 3.2 For $\varepsilon>0, K \geq 3$ the variational problem

$$
\min \left\{E_{\varepsilon}(v): v \in \mathcal{B}_{K}\right\}
$$

attains its minimum.

Proof. The proof follows the same lines as the corresponding existence proof of Kohn and Müller [13]. For completeness we briefly sketch the argument. 
Fix $\varepsilon>0$. Let $v^{(k)} \subset \mathcal{B}_{K}$ be a minimising sequence for $E_{\varepsilon}$. Then $v^{(k)}$ is bounded in $H^{1}$ and $\nabla \nabla v^{(k)}$ is bounded in $\mathcal{M}$ (the space of Radon measures). Passing to a subsequence one has

$$
\begin{aligned}
& v^{(k)} \rightarrow v \quad \text { in } H^{1}\left(R_{L, H}\right), \\
& \nabla \nabla v^{(k) \stackrel{*}{*}} \nabla \nabla v \quad \text { in } \mathcal{M} .
\end{aligned}
$$

By standard lower semicontinuity results

$$
E_{\varepsilon}(v) \leq \liminf _{k \rightarrow \infty} E_{\varepsilon}\left(v^{(k)}\right)
$$

The only subtle point is to show that $v \in \mathcal{B}_{K}$. By assumption $\nabla v^{(k)}$ is bounded in $L^{\infty}$ so that $\nabla \nabla v^{(k)}$ is bounded in $\mathcal{M} \cap W^{-1, \infty}$. By Murat's lemma (see [15]) $\nabla \nabla v^{(k)}$ lies in a compact set of $H^{-1}$. This implies that $\nabla v^{(k)} \rightarrow \nabla v$ in $L_{l o c}^{2}\left(R_{L, H}\right)$ (as was proved in [13] in a lemma). Passing to a further subsequence one sees that that $\nabla v \leq K$ a.e. We also have that $v=J_{1} x$ by standard trace theorems. Hence $v \in \mathcal{B}_{K}$ and the proposition is proved.

We finish the proof of Theorem 3.1 in three steps. First, we define a sequence of functions $v^{(1)}, v^{(2)}, \ldots \in \mathcal{B}_{K}$ by infinite sequential lamination. Second, we define $v$ by fixing $k=k(\varepsilon)$ and setting $v=v^{(k(\varepsilon))}$ where the integer $k(\varepsilon)$ is chosen such that $v^{(k(\varepsilon))}$ satisfies the following Lemma.

Lemma 3.1 For every constant $\sigma$ with $0<\sigma<\sqrt{2 \log 2}$ there exist constants $C>0$ and $\varepsilon_{0}>0$ such that for all $0<\varepsilon<\varepsilon_{0}$ and $K \geq 3$

$$
E_{\varepsilon}\left(v^{(k(\varepsilon))}\right) \leq C \exp \left(-\sigma \sqrt{\log \frac{1}{\varepsilon}}\right)
$$

Third, we define $u \in \mathcal{A}_{K}$ by convolution of $v$ with a mollifier kernel and show that the resulting $u$ satisfies an estimate analog to (3.4).

Step 1. The sequence $v^{(i)}$

We define the functions $v^{(i)}$ recursively by sequential lamination. In each refinement step triangulation on a smaller scale is introduced at part of the boundary to make sure that $v^{(i)} \in W^{1, \infty}\left(R_{L, H}\right)$ and that the boundary conditions are satisfied. We make this construction 
explicit and calculate the corresponding energy. Let us begin by defining the zig-zag function $z$ as follows:

$$
z(s)=\left\{\begin{array}{ll}
s & \text { if } 0 \leq s \leq \frac{1}{2} \\
1-s & \text { if } \frac{1}{2} \leq s \leq 1
\end{array}\right\}=\min \{s, 1-s\} \quad \text { for } 0 \leq s \leq 1
$$

and $z$ is continued 1-periodically onto the real axis. Then, for a positive integer $n_{1}$ and $l_{1}=H / n_{1}$, we introduce

$$
v^{(1)}(x)= \begin{cases}J_{1} x+l_{1} z\left(x_{2} / l_{1}\right)\left(\begin{array}{l}
0 \\
2
\end{array}\right) & \text { if } l_{1} z\left(x_{2} / l_{1}\right) \leq x_{1} \leq L-l_{1} z\left(x_{2} / l_{1}\right), \\
J_{1} x+x_{1}\left(\begin{array}{l}
0 \\
2
\end{array}\right) & \text { if } 0 \leq x_{1} \leq l_{1} z\left(x_{2} / l_{1}\right) \\
J_{1} x+\left(L-x_{1}\right)\left(\begin{array}{l}
0 \\
2
\end{array}\right) & \text { if } L-l_{1} z\left(x_{2} / l_{1}\right) \leq x_{1} \leq L\end{cases}
$$

see Figure 2. The value for $n_{1}$ will be chosen later. Note that $v^{(1)}$ is an example of single lamination. This means that the domain $R_{L, H}$ is divided into periodically alternating layers of width $H /\left(2 n_{1}\right)$ where $\nabla v^{(1)}$ takes on the values $A$ and $J_{2}$, respectively. This is expressed by the first equation in the definition of $v^{(1)}$. Furthermore, some triangulation near the left and right boundaries of $R_{L, H}$ is required to make sure that $v^{(1)}$ satisfies the boundary conditions. This is done in the last two equations. The function $v^{(1)}$ is piecewise linear with $\nabla v^{(1)}$ attaining (only) the values $A, J_{2},\left(\begin{array}{ll}1 & 0 \\ 2 & 1\end{array}\right)$, or $\left(\begin{array}{rr}1 & 0 \\ -2 & 1\end{array}\right)$.

We now calculate the energy $E_{\varepsilon}\left(v^{(1)}\right)$ is as follows. The elastic energy consists of three terms corresponding to the last three values of $\nabla v^{(1)}$, respectively, the main contribution coming from the set where $\nabla v^{(1)}=J_{2}$. The surface energy is " $\varepsilon \times$ (total length of interfaces) $\times$ (change of $\left.\nabla v^{(1)}\right)$ ". Its main contribution comes from the interfaces created by layering between the gradients $A$ and $J_{2}$ plus a smaller contribution coming from triangulation near the left and right boundaries. To estimate the energy of $v^{(1)}$ it is important to know that by 
the continuity of $W$ there exist constants $c, C>0$ such that

$$
\begin{gathered}
c \leq \max \left\{J_{1}, J_{2}, J_{3}, J_{4}, W\left(\left(\begin{array}{ll}
1 & 0 \\
2 & 1
\end{array}\right)\right), W\left(\left(\begin{array}{cc}
1 & 0 \\
-2 & 1
\end{array}\right)\right),\right. \\
W\left(\left(\begin{array}{ll}
-1 & 0 \\
2 & -1
\end{array}\right)\right), W\left(\left(\begin{array}{ll}
-1 & 0 \\
-2 & -1
\end{array}\right)\right), W\left(\left(\begin{array}{ll}
1 & 2 \\
0 & -1
\end{array}\right)\right), \\
\left.W\left(\left(\begin{array}{ll}
1 & -2 \\
0 & -1
\end{array}\right)\right), W\left(\left(\begin{array}{ll}
-1 & 2 \\
0 & 1
\end{array}\right)\right), W\left(\left(\begin{array}{cc}
-1 & -2 \\
0 & 1
\end{array}\right)\right)\right\} \leq C .
\end{gathered}
$$

Furthermore, bear in mind that the width of the layers and the sides of the triangles near the boundary are both of order $l_{1}$, up to a multiplicative constant. Therefore

$$
\begin{aligned}
& E_{\varepsilon}\left(v^{(1)}\right)=W\left(J_{2}\right)\left|\left\{\nabla v^{(1)}=J_{2}\right\}\right|+W\left(\left(\begin{array}{cc}
1 & 0 \\
2 & 1
\end{array}\right)\right)\left|\left\{\nabla v^{(1)}=\left(\begin{array}{cc}
1 & 0 \\
2 & 1
\end{array}\right)\right\}\right| \\
& +W\left(\left(\begin{array}{cc}
1 & 0 \\
-2 & 1
\end{array}\right)\right)\left|\left\{\nabla v^{(1)}=\left(\begin{array}{cc}
1 & 0 \\
-2 & 1
\end{array}\right)\right\}\right| \\
& +\varepsilon\left(\# \text { jumps of } \nabla v^{(1)} \text { between } A \text { and } J_{2}\right) \times \\
& \times(\text { length of interfaces }) \times\left(\text { change of } \nabla v^{(1)}\right) \\
& +\varepsilon \text { (\# triangles near left and right boundaries }) \times \\
& \times\left(\text { length of triangle sides which lie inside } R_{L, H}\right) \times \\
& \times\left(\text { change of } \nabla v^{(1)}\right) \\
& \leq C\left\{\left(\frac{1}{2} L H-\frac{1}{4} l_{1} H+\frac{1}{2} l_{1} H\right)\right. \\
& \left.+\varepsilon\left(\left(\frac{H}{l_{1}}-1\right) L+\frac{H}{l_{1}}\left(L-l_{1}\right)\right)+\varepsilon\left(2 \frac{H}{l_{1}} l_{1} \sqrt{2}\right)\right\} \\
& \leq C\left(L H+l_{1} H+\varepsilon \frac{L H}{l_{1}}\right)+\mathcal{O}(\varepsilon) \quad \text { as } \varepsilon \rightarrow 0 \\
& \leq C\left(L H+\varepsilon \frac{L H}{l_{1}}\right) \quad \text { as } \varepsilon \rightarrow 0 \quad \text { by }(3.7) \text {. }
\end{aligned}
$$


Furthermore, these estimates are sharp up to a multiplicative constant. Now we present the infinite sequential lamination in a rigorous setting. To get geometric intuition of the construction the reader should study the relatively complex formal framework in connection with Figures 3 and 4 . To begin with, we have to introduce some notation. Define

$$
\begin{aligned}
l_{1} & =H / n_{1}, \\
l_{2} & =\left(L-l_{1}\right) / n_{2}, \\
l_{i+1} & =\left(l_{i-1} / 2-l_{i}\right) / n_{i+1}, \quad i=2,3, \ldots
\end{aligned}
$$

where the integers $n_{1}, n_{2}, \ldots$ will be chosen later.

We are studying a microstructure with multiple length scales which are denoted by $l_{i}$. The following is a rigorous formulation of this fact.

Assumption: Assume that

$$
\frac{l_{i+1}}{l_{i}} \rightarrow 0 \quad \text { as } \varepsilon \rightarrow 0 \quad \text { for } i=1,2, \ldots
$$

and

$$
\frac{H}{l_{1}} \rightarrow 0 \quad \text { as } \varepsilon \rightarrow 0 .
$$

We define now sets $[0, L] \supset X_{1} \supset X_{2} \supset \ldots$ and $[0, H] \supset Y_{1} \supset Y_{2} \supset \ldots$ by recursion.

Definition 3.1 Define $X_{i}, i=1,2, \ldots$ as follows:

i) $X_{1}=[0, L]$.

ii) $X_{2}=\left\{x \in[0, L]: \operatorname{dist}(x,\{0, L\}) \geq l_{1} / 2\right.$,

iii) $\left.X_{2 k+1}=\left\{x \in X_{2 k}: \operatorname{dist}\left(x, \sup \left\{t \notin X_{2 k}: t \leq x\right\}\right) / l_{2 k}\right)_{\bmod 1} \in[1 / 2,1]\right\}, \quad k=1,2, \ldots$

iv) $X_{2 k}=\left\{x \in X_{2 k-1}: \operatorname{dist}\left(x,[0, L] \backslash X_{2 k-1}\right) \geq l_{2 k-1} / 2\right\}, \quad k=2,3 \ldots$

Set $s_{2 k}(x)=\sup \left\{t \notin X_{2 k}: t \leq x\right\}$.

Define $Y_{i}, i=1,2, \ldots$ as follows:

i) $Y_{1}=[0, H]$.

ii) $\left.Y_{2 k}=\left\{y \in Y_{2 k-1}: \operatorname{dist}\left(y, \sup \left\{t \notin Y_{2 k}: t \leq y\right\}\right) / l_{2 k-1}\right)_{\bmod 1} \in[1 / 2,1]\right\}, \quad k=1,2, \ldots$

iii) $Y_{2 k+1}=\left\{y \in Y_{2 k}: \operatorname{dist}\left(y,[0, H] \backslash Y_{2 k}\right) \geq l_{2 k} / 2\right\}, \quad k=1,2, \ldots$

Set $s_{2 k-1}(y)=\sup \left\{t \notin Y_{2 k-1}: t \leq y\right\}$. 
Remark: We have for example

$$
\begin{aligned}
X_{2} & =\left[l_{1} / 2, L-l_{1} / 2\right], \\
X_{3} & =\bigcup_{i_{2}=1}^{n_{2}}\left[l_{1} / 2+\left(i_{2}-1 / 2\right) l_{2}, l_{1} / 2+i_{2} l_{2}\right], \\
X_{4} & =\bigcup_{i_{2}=1}^{n_{2}}\left[l_{1} / 2+\left(i_{2}-1 / 2\right) l_{2}+l_{3} / 2, l_{1} / 2+i_{2} l_{2}-l_{3} / 2\right], \\
Y_{2} & =\bigcup_{i_{1}=1}^{n_{1}}\left[\left(i_{1}-1 / 2\right) l_{1}, i_{1} l_{1}\right], \\
Y_{3} & =\bigcup_{i_{1}=1}^{n_{1}}\left[\left(i_{1}-1 / 2\right) l_{1}+l_{2} / 2, i_{1} l_{1}-l_{2} / 2\right], \\
Y_{4} & =\bigcup_{i_{1}=1}^{n_{1}} \bigcup_{i_{3}=1}^{n_{3}}\left[\left(i_{1}-1 / 2\right) l_{1}+l_{2} / 2+\left(i_{3}-1 / 2\right) l_{3},\left(i_{1}-1 / 2\right) l_{1}+l_{2} / 2+i_{3} l_{3}\right] .
\end{aligned}
$$

Using the sets from Definition 3.1 we are now in a position to define the functions $v^{(2 k)}$ and $v^{(2 k+1)}$ recursively:

$$
\begin{aligned}
& v^{(2 k)}(x)=v^{(2 k-1)}(x)+(-1)^{k-1}\left(\begin{array}{l}
2 \\
0
\end{array}\right) \times \\
& \times \begin{cases}0 & \text { if } x_{1} \notin X_{2 k} \text { or } x_{2} \notin Y_{2 k} \\
l_{2 k} z\left(\left(x_{1}-s_{2 k}\left(x_{1}\right)\right) / l_{2 k}\right) & \text { if } x_{1} \in X_{2 k}, x_{2} \in Y_{2 k}, \text { and } \\
& s_{2 k-1}\left(x_{2}\right)+l_{2 k} z\left(\left(x_{1}-s_{2 k}\left(x_{1}\right)\right) / l_{2 k}\right) \leq x_{2} \leq \\
& \leq s_{2 k-1}\left(x_{2}\right)+l_{2 k-1} / 2-l_{2 k} z\left(\left(x_{1}-s_{2 k}\left(x_{1}\right)\right) / l_{2 k}\right) \\
x_{2}-s_{2 k-1}\left(x_{2}\right) & \text { if } x_{1} \in X_{2 k}, x_{2} \in Y_{2 k}, \text { and } \\
s_{2 k-1}\left(x_{2}\right)+l_{2 k-1} / 2-x_{2} & \text { if } x_{1} \in X_{2 k}, x_{2} \in Y_{2 k}, \text { and } \\
& s_{2 k-1}\left(x_{2}\right)+l_{2 k-1} / 2-l_{2 k} z\left(\left(x_{1}-s_{2 k}\left(x_{1}\right)\right) / l_{2 k}\right) \leq x_{2} \leq \\
& \leq s_{2 k-1}\left(x_{2}\right)+l_{2 k-1} / 2\end{cases}
\end{aligned}
$$

and

$$
v^{(2 k+1)}(x)=v^{(2 k)}(x)+(-1)^{k}\left(\begin{array}{l}
0 \\
2
\end{array}\right) \times
$$




$$
\times \begin{cases}0 & \text { if } x_{1} \notin X_{2 k+1} \text { or } x_{2} \notin Y_{2 k+1} \\ l_{2 k+1} z\left(\left(x_{2}-s_{2 k+1}\left(x_{2}\right)\right) / l_{2 k+1}\right) & \text { if } x_{1} \in X_{2 k+1}, x_{2} \in Y_{2 k+1}, \text { and } \\ & s_{2 k}\left(x_{1}\right)+l_{2 k+1} z\left(\left(x_{2}-s_{2 k+1}\left(x_{2}\right)\right) / l_{2 k+1}\right) \leq x_{1} \leq \\ & \leq s_{2 k}\left(x_{1}\right)+l_{2 k} / 2-l_{2 k+1} z\left(\left(x_{2}-s_{2 k+1}\left(x_{2}\right)\right) / l_{2 k+1}\right) \\ x_{1}-s_{2 k}\left(x_{1}\right) & \text { if } x_{1} \in X_{2 k+1}, x_{2} \in Y_{2 k+1}, \text { and } \\ & s_{2 k}\left(x_{1}\right) \leq x_{1} \\ & \leq s_{2 k}\left(x_{1}\right)+l_{2 k+1} z\left(\left(x_{2}-s_{2 k+1}\left(x_{2}\right)\right) / l_{2 k+1}\right) \\ & \text { if } x_{1} \in X_{2 k+1}, x_{2} \in Y_{2 k+1}, \text { and } \\ s_{2 k}\left(x_{1}\right)+l_{2 k} / 2-x_{1} & s_{2 k}\left(x_{1}\right)+l_{2 k} / 2-l_{2 k+1} z\left(\left(x_{2}-s_{2 k+1}\left(x_{2}\right)\right) / l_{2 k+1}\right) \\ & \leq x_{1} \leq \leq s_{2 k}\left(x_{1}\right)+l_{2 k} / 2 .\end{cases}
$$

Note that in each of the previous two formulae the second line describes the new lamination step. The last two lines correspond to triangulation which is required to make the function continuous. We can now calculate the general formula for $E_{\varepsilon}\left(v^{(i)}\right), i=1,2, \ldots$ by induction. Note that this can be done in a unified way and no distinction has to be made between odd and even values for $i$. Let us first calculate the difference $E_{\varepsilon}\left(v^{(i+1)}\right)-E_{\varepsilon}\left(v^{(i)}\right)$. Going from $v^{(i)}$ to $v^{(i+1)}$ refinements have to be made on

$$
\begin{aligned}
\#_{i} & =\frac{H}{l_{1}} \frac{L-l_{1}}{l_{2}} \frac{l_{1} / 2-l_{2}}{l_{3}} \cdots \frac{l_{i-2} / 2-l_{i-1}}{l_{i}} \\
& =\frac{1}{2^{i-2}} L H\left(1-\frac{l_{1}}{L}\right)\left(1-\frac{2 l_{2}}{l_{1}}\right) \cdots\left(1-\frac{2 l_{i-1}}{l_{i-2}}\right) \frac{1}{l_{i-1} l_{i}} \\
& =\frac{1}{2^{i-2}} \frac{L H}{l_{i-1} l_{i}} \mathcal{P}_{i}
\end{aligned}
$$

rectangles whose sides are of the length $l_{i} / 2$ and $l_{i-1} / 2-l_{i}$, respectively. Here

$$
\mathcal{P}_{i}=\left(1-\frac{l_{1}}{L}\right) \cdot\left(1-\frac{2 l_{2}}{l_{1}}\right) \cdots\left(1-\frac{2 l_{i}}{l_{i-1}}\right), \quad i=1,2, \ldots
$$

The upper bound of the contribution (which is sharp up to multiplicative constant) of each of these rectangles to the elastic energy changes from

$$
C\left(l_{i-1} / 2-l_{i}\right)\left(l_{i} / 2\right)
$$


to

$$
\begin{aligned}
& C\left\{\left(l_{i-1} / 4-l_{i} / 2\right)\left(l_{i} / 2-l_{i+1} / 2\right)\right. \\
& \left.+\left(l_{i-1} / 2-l_{i}\right)\left(l_{i+1} / 2\right)\right\}
\end{aligned}
$$

the last expression consisting of contributions from lamination and triangulation, respectively. In each of these rectangles new interfaces have to be created, and their total length (per rectangle) is

$$
2 \sqrt{2}\left(l_{i-1} / 2-l_{i}\right)+\frac{l_{i-1} / 2-l_{i}}{l_{i+1}}\left(l_{i} / 2-l_{i+1}\right)+\left(\frac{l_{i-1} / 2-l_{i}}{l_{i+1}}+1\right) \frac{1}{2} l_{i} \leq C \frac{l_{i-1} l_{i}}{l_{i+1}} .
$$

Therefore the total contribution of these rectangles to the upper bound for the total energy changes from

$$
E_{\varepsilon}\left(v^{(i)}\right) \leq C L H \frac{1}{2^{i-3}} \mathcal{P}_{i-1}
$$

to

$$
E_{\varepsilon}\left(v^{(i+1)}\right) \leq C\left(L H \frac{1}{2^{i-2}} \mathcal{P}_{i}+L H \frac{1}{2^{i-3}} \mathcal{P}_{i} \frac{l_{i+1}}{l_{i}}+\varepsilon L H \frac{1}{2^{i}} \mathcal{P}_{i-1} \frac{1}{l_{i}}\right)
$$

Note that the last two expressions are sharp up to a multiplicative constant. Using the expansion for $E_{\varepsilon}\left(v^{(1)}\right)$ and the previous formulae it follows by induction that

$$
E_{\varepsilon}\left(v^{(k)}\right) \leq C F_{\varepsilon}\left(v^{(k)}\right)+\mathcal{O}(\varepsilon) \quad \text { as } \varepsilon \rightarrow 0
$$

where

$$
\begin{aligned}
F_{\varepsilon}\left(v^{(k)}\right)= & \frac{1}{2^{k}} L H+L H\left\{\frac{l_{1}}{L}+\frac{l_{2}}{l_{1}}+\frac{l_{3}}{2 l_{2}}+\ldots+\frac{l_{k}}{2^{k-2} l_{k-1}}\right\} \\
& +\varepsilon L H\left\{\frac{1}{l_{1}}+\frac{1}{2 l_{2}}+\ldots+\frac{1}{2^{k-1} l_{k}}\right\}+\mathcal{O}(\varepsilon) \quad \text { as } \varepsilon \rightarrow 0 .
\end{aligned}
$$

All these estimates are sharp up to a multiplicative constant. Note that the expression for $F_{\varepsilon}\left(v^{(k)}\right)$ was obtained from $E_{\varepsilon}\left(v^{(k)}\right)$ by neglecting the factors $\left(1-l_{1} / L\right)$ and $\left(1-2 l_{i+1} / l_{i}\right), i=$ $1,2, \ldots$ which are smaller than 1 and converge to 1 as $\varepsilon \rightarrow 0$.

Step 2. The function $v$

We now choose the integer $k=k(\varepsilon)$ in such a way that for $v=v^{(k(\varepsilon))}$ the asymptotic behaviour of $F_{\varepsilon}(v)$ as $\varepsilon \rightarrow 0$ will be optimal. The following lemma states an upper bound for the asymptotic behaviour of $F_{\varepsilon}\left(v^{(k)}\right)$ as $\varepsilon \rightarrow 0$. 
Lemma 3.2 For every $\sigma$ with $0<\sigma<\sqrt{2 \log 2}$ there exist numbers $C>0$ and $\varepsilon_{0}>0$ such that

$$
\inf \left\{F_{\varepsilon}\left(v^{(k)}\right): k=1,2, \ldots\right\} \leq C \exp \left(-\sigma \sqrt{\log \frac{1}{\varepsilon}}\right)
$$

for $0<\varepsilon<\varepsilon_{0}$.

Proof. Set

$$
l_{i}=\varepsilon^{\alpha_{i}}
$$

where $\alpha_{i}=\alpha_{i}(\varepsilon)$. Inserting these terms in the expansion for $F_{\varepsilon}\left(v^{(k)}\right)$ implies

$$
\begin{aligned}
F_{\varepsilon}\left(v^{(k)}\right) \leq & C\left\{\frac{1}{2^{k}}+\varepsilon^{\alpha_{1}}+\varepsilon^{\alpha_{2}-\alpha_{1}}+\frac{1}{2} \varepsilon^{\alpha_{3}-\alpha_{2}}+\ldots+\frac{1}{2^{k-2}} \varepsilon^{\alpha_{k}-\alpha_{k-1}}\right. \\
& \left.+\varepsilon^{1-\alpha_{1}}+\frac{1}{2} \varepsilon^{1-\alpha_{2}}+\ldots+\frac{1}{2^{k-1}} \varepsilon^{1-\alpha_{k}}\right\}
\end{aligned}
$$

Let us now first derive condition for $\sigma$ which is necessary for (3.8) to hold. Afterwards we show that a slightly weakened condition is also sufficient.

Because every term must be smaller than $C \exp (-\sigma \sqrt{\log (1 / \varepsilon)})$ by taking logarithms we obtain that the following conditions are necessary for (3.8):

$$
\begin{aligned}
\frac{\sigma}{\log 2} & \leq \frac{k}{\rho}+\frac{C}{\rho}, \\
\sigma & \leq \rho \alpha_{1}+\frac{C}{\rho}, \\
\sigma & \leq \frac{i}{\rho} \log 2+\rho\left(\alpha_{i+1}-\alpha_{i}\right)+\frac{C}{\rho}, \quad i=1,2, \ldots, k-1 \\
\sigma & \leq \frac{i}{\rho} \log 2+\rho\left(1-\alpha_{i}\right)+\frac{C}{\rho}, \quad i=1,2, \ldots, k
\end{aligned}
$$

for $i=1,2, \ldots$ where $\rho=\rho(\varepsilon)=\sqrt{\log (1 / \varepsilon)}$. Adding (3.11) and (3.12) for $i=1,2, \ldots, k-1$ we deduce

$$
\alpha_{k} \geq \sigma \frac{k}{\rho}-\frac{\log 2}{2}\left(\frac{k}{\rho}\right)^{2}-C \frac{k}{\rho^{2}}
$$

where $C$ is independent of $k$. It follows from (3.13) for $i=k$ that

$$
\alpha_{k} \leq 1-\frac{\sigma}{\rho}+\frac{k}{\rho^{2}} \log 2+\frac{C}{\rho^{2}}
$$


where $C$ is independent of $k$. Therefore

$$
\sigma \frac{k}{\rho}-\frac{\log 2}{2}\left(\frac{k}{\rho}\right)^{2} \leq 1+C \frac{1}{\rho}\left(1+\frac{k}{\rho}\right)
$$

where $C$ is independent of $k$. The left-hand side of (3.16) attains its minimum if

$$
\frac{k}{\rho}=\frac{\sigma}{\log 2}
$$

and has the value $\sigma^{2} /(2 \log 2)$. Choose $k=[\rho \sigma / \log 2]$ where [.] is the integer function. Inserting this into (3.16) implies that

$$
\frac{\sigma^{2}}{2 \log 2} \leq 1+o(1)
$$

as $\varepsilon \rightarrow 0$. Therefore, a necessary condition for the solvability of system (3.10)-(3.13) is

$$
\sigma \leq \sqrt{2 \log 2}
$$

For $\sigma<\sqrt{2 \log 2}$ we are now giving a solution of (3.8). We again make the substitution (3.9). Furthermore, we set

$$
\alpha_{i}=i \mu-\frac{(i-1) i}{2} \tau, \quad i=1,2, \ldots, k
$$

where the positive real numbers $\mu$ and $\tau$ are still to be determined. This implies

$$
\begin{aligned}
F_{\varepsilon}\left(v^{(k)}\right) \leq & C\left(\frac{1}{2^{k}}+\left\{\varepsilon^{\mu}+2 \varepsilon^{\mu} \sum_{i=1}^{k-1}\left(\frac{\varepsilon^{-\tau}}{2}\right)^{i}\right\}\right. \\
& \left.+\frac{1}{2^{k-1}} \varepsilon^{1-k \mu+(k-1) k \tau / 2}\left\{\sum_{i=0}^{k-1} 2^{i} \varepsilon^{i \mu-((k-1) k-(k-i-1)(k-i)) \tau / 2}\right\}\right) \\
\leq & C\left(\frac{1}{2^{k}}+\left\{\varepsilon^{\mu}+2 \varepsilon^{\mu} \sum_{i=1}^{k-1}\left(\frac{\varepsilon^{-\tau}}{2}\right)^{i}\right\}\right. \\
& \left.+\frac{1}{2^{k-1}} \varepsilon^{1-k \mu+(k-1) k \tau / 2}\left\{\sum_{i=0}^{k-1}\left(2 \varepsilon^{\mu-k \tau}\right)^{i}\right\}\right) .
\end{aligned}
$$

Again we set $\rho(\varepsilon)=\sqrt{\log (1 / \varepsilon)}$. Choose the parameters as follows:

$$
\begin{aligned}
k(\rho) & =\left[\frac{\sigma \rho}{\log 2}\right] \\
\mu(\rho) & =\frac{\sigma}{\rho} \\
\tau(\rho) & <\frac{\log 2}{\rho^{2}} .
\end{aligned}
$$


Furthermore, by choosing $\tau(\rho)$ such that $\tau(\rho) \rho^{2}$ is sufficiently close to log 2 , we have

$$
1-k \mu+(k-1) k \frac{\tau}{2}=1-\frac{\sigma^{2}}{\log 2}+\frac{\sigma^{2} \rho^{2}}{2(\log 2)^{2}} \tau+\mathcal{O}\left(\frac{1}{\rho}\right)>0
$$

for all $\rho>\rho_{0}$ where $\rho_{0}>0$ is fixed. The equations (3.20)-(3.23) imply

$$
\begin{gathered}
\frac{1}{2^{k}} \leq C \exp (-\sigma \sqrt{\log (1 / \varepsilon)}), \\
\varepsilon^{\mu}=\exp (-\sigma \sqrt{\log (1 / \varepsilon)}) \\
\mu-k \tau>0 \\
1-k \mu+(k-1) k \tau / 2>0 .
\end{gathered}
$$

Therefore we see from (3.19) that the conditions (3.20)-(3.23) are sufficient for the estimate (3.8). This concludes the proof of Lemma 3.2.

Step 3. The function $u$

Now we go back to the original variational problem (3.1). We define test functions $u^{(k)}=$ $u^{(k(\varepsilon))}$ by convolution of $v^{(k)}=v^{(k(\varepsilon))}$ with a mollifier kernel $\Phi_{\varepsilon}$. In the sequel we omit the $\operatorname{argument} \varepsilon$ of $k(\varepsilon)$ where this does not cause confusion. That is we introduce

$$
u^{(k)}(x)=\int_{R_{L, H}} v^{(k)}(s) \Phi_{\varepsilon}(|x-s|) d s
$$

(in each component) where

$$
\begin{aligned}
\Phi_{\varepsilon}(t) & =\frac{1}{\varepsilon^{2}} \Phi(t / \varepsilon) \quad \text { for all } t \in R \\
\Phi & \in \mathcal{C}_{0}^{\infty}([0,1]) \\
\int_{B(0,1)} \Phi(x) d x & =1 \\
0 & \leq \Phi \leq 1
\end{aligned}
$$

with $B(0,1)$ the two dimensional (closed) unit ball. It follows that (again in each component)

$$
\begin{aligned}
\nabla u^{(k)}(x) & =\int_{R_{L, H}} \nabla v^{(k)}(s) \Phi_{\varepsilon}(|x-s|) d s \\
\nabla \nabla u^{(k)}(x) & =\int_{R_{L, H}} \nabla v^{(k)}(s) \nabla_{x} \Phi_{\varepsilon}(|x-s|) d s \\
& \left.=-\sum_{\nu} \int_{\Omega_{\nu}^{(k)}} \nabla v^{(k)}(s) \nabla_{s} \Phi_{\varepsilon}|x-s|\right) d s \\
& =\sum_{\nu} \int_{\partial \Omega_{\nu}^{(k)}} \nabla v^{(k)}(s(l)) \Phi_{\varepsilon}(|x-s(l)|) n d l
\end{aligned}
$$


where $\Omega_{\nu}^{(k)}$ are the subsets of $R_{L, H}$ where $\nabla v^{(k)}$ is constant, $n$ is the interior unit normal vector on $\partial \Omega_{\nu}^{(k)}, d l$ is the one-dimensional Hausdorff measure on $\partial \Omega_{\nu}^{(k)}$. Note that $\nu$ is finite for fixed $k(\varepsilon)$. The elastic energy of $u^{(k)}$ can be estimated as follows.

$$
\begin{aligned}
\int_{R_{L, H}} W\left(\nabla u^{(k)}\right) d x= & \int_{R_{L, H}} W\left(\nabla v^{(k)}\right) d x \\
& +\left(\int_{R_{L, H}} W\left(\nabla u^{(k)}\right) d x-\int_{R_{L, H}} W\left(\nabla v^{(k)}\right) d x\right) \\
\leq & C \exp \left(-\sigma \sqrt{\log \frac{1}{\varepsilon}}\right)+C \operatorname{meas}\left\{x \in R_{L, H}: \nabla v^{(k)} \neq \nabla u^{(k)}\right\} \\
\leq & C \exp \left(-\sigma \sqrt{\log \frac{1}{\varepsilon}}\right)+\varepsilon 2 C\left|\Gamma^{(k)}\right| \\
\leq & C \exp \left(-\sigma \sqrt{\log \frac{1}{\varepsilon}}\right)
\end{aligned}
$$

by (3.8). We have used the notation $\Gamma^{(k)}=\bigcup_{\nu} \partial \Omega_{\nu}^{(k)}$. It is clear that the estimate for $\left\{x \in R_{L, H}: \nabla v^{(k)} \neq \nabla u^{(k)}\right\}$ is correct if $\Gamma^{(k)}$ is a straight line. Furthermore, note that near corners of $\Gamma^{(k)}$ the estimate for $\left\{x \in R_{L, H}: \nabla v^{(k)} \neq \nabla u^{(k)}\right\}$ is correct. Note also that the same is true if three or more lines meet in one point. (In both cases there is some "overlap".) With (3.8) and since $\varepsilon\left|\Gamma^{(k)}\right|$ is the contribution of the surface energy to $F_{\varepsilon}\left(v^{(k)}\right)$ the last inequality above follows. Since $\varepsilon\left|\Gamma^{(k)}\right|$ is the contribution of the surface energy to $F_{\varepsilon}\left(v^{(k)}\right)$ the last inequality follows from (3.8). Let us now estimate the surface energy. By the divergence theorem

$$
\begin{aligned}
& \varepsilon^{2}\left\|\nabla \nabla u^{(k)}\right\|^{2}= \\
& =\varepsilon^{2} \int_{R_{L, H}} \sum_{\iota, \kappa, \xi=1}^{2}\left(\int_{\partial \Omega_{\nu}^{(k)}} \partial_{\kappa} v_{\iota}^{(k)}(s(l)) \Phi_{\varepsilon}(|x-s(l)|) n_{\xi} d l\right)^{2} d x \\
& =\varepsilon^{2} \int_{R_{L, H}} \sum_{\iota, \kappa, \xi=1}^{2}\left(\int_{\Gamma^{(k)}}\left(\partial_{\kappa} v_{\iota+}^{(k)}(s(l))-\partial_{\kappa} v_{\iota-}^{(k)}(s(l))\right) \times\right. \\
& \left.\quad \times \Phi_{\varepsilon}(|x-s(l)|) n_{\xi} d l\right)^{2} d x
\end{aligned}
$$

where $\partial_{\kappa} v_{\iota \pm}^{(k)}$ denote the limits of $\partial_{\kappa} v_{\iota}^{(k)}(s)$ as $s$ approaches the interface $\Gamma$ (from either side). Note that $\Gamma^{(k)}$ belongs to two sets of the type $\partial \Omega_{\nu}^{(k)}$ unless $\Gamma^{(k)} \subset \partial R_{L, H}$. Because of

$$
\left\|\partial_{\kappa} v_{\iota}^{(k)}\right\|^{2} \leq C
$$


with $C$ independent of $k$ it follows by Fubini's theorem that

$$
\varepsilon^{2}\left\|\nabla \nabla u^{(k)}\right\|^{2} \leq C \varepsilon^{2} \int_{\delta=0}^{\varepsilon} \int_{\Gamma_{\delta}^{(k)}}\left(\int_{\Gamma^{(k)}} \Phi_{\varepsilon}(|x-s(l)|) d l\right)^{2} d \bar{l} d \delta
$$

where

$$
\Gamma_{\delta}^{(k)}=\left\{x: \operatorname{dist}\left(x, \Gamma^{(k)}\right)=\delta\right\}
$$

The smallest length scale of the length of the interfaces of $\Gamma^{(k)}$ is

$$
l_{k}=\varepsilon^{\sigma^{2} /(2 \log 2)}
$$

If $\sigma<\sqrt{2 \log 2}$, then $l_{k} \gg \varepsilon$ as $\varepsilon \rightarrow 0$. Furthermore, by the construction of the microstructure

$$
\operatorname{dist}\left(\Gamma_{i}, \Gamma_{i+2}\right) \geq \frac{l_{i+1}}{2 \sqrt{2}} \quad i=0,1, \ldots, k-2 .
$$

Therefore the number of interfaces having nonempty intersection with an $\varepsilon$-neighbourhood $B(x, \varepsilon)$ of $x$ is bounded uniformly in $x$ and $\varepsilon$. (We have used the notation

$$
\left.B(x, r)=\left\{s \in R^{2}:|x-s| \leq r\right\} .\right)
$$

Using the properties of the mollifier $\Phi_{\varepsilon}$ this implies

$$
\begin{aligned}
& \left(\int_{\Gamma^{(k)}} \Phi_{\varepsilon}(|x-s(l)|) d l\right)^{2} \leq \\
& \leq \frac{\|\Phi\|_{\infty}^{2}}{\varepsilon^{4}}\left(\int_{\Gamma^{(k)} \cap B(x, \varepsilon)} 1 d l\right)^{2} \\
& \quad \leq C \frac{\|\Phi\|_{\infty}^{2}}{\varepsilon^{2}} .
\end{aligned}
$$

Since $\left|\Gamma_{\delta}^{(k)}\right| \leq 2\left|\Gamma^{(k)}\right|$ we obtain from (3.29)

$$
\varepsilon^{2}\left\|\nabla \nabla u^{(k)}\right\|^{2} \leq \varepsilon C\left|\Gamma^{(k)}\right|
$$

With (3.8) and since $\varepsilon\left|\Gamma^{(k)}\right|$ is the contribution of the surface energy to $F_{\varepsilon}\left(v^{(k)}\right)$ we finally obtain

$$
\varepsilon^{2}\left\|\nabla \nabla v^{(k)}\right\|^{2} \leq C \exp \left(-\sigma \sqrt{\log \frac{1}{\varepsilon}}\right) .
$$

This concludes the proof of Theorem 3.1. 


\section{Discussion}

The achievement of the paper is twofold.

First we have presented a result of a general nature, which gives a restriction for any possible microstructure, namely that its Young measure is unique and homogeneous.

Second an infinitely sequentially-laminated microstructure has been constructed. The parameters such as length scales and the number of layerings are chosen to depend on $\varepsilon$ so as to achieve an optimal asymptotic behaviour of the corresponding energy. We do, however, not show that this is the only microstructure giving such an asymptotic behaviour (or that there is no microstructure that has an even better asymptotic behaviour). But we expect that our microstructure is optimal in this sense. This is also supported by the numerical results in [8].

Although the configuration under consideration seems special we do expect similar behaviour for a wide range of variational problems leading to infinitely sequentially-laminated microstructure. We would like to conclude with the remark that the microstructure involved is very complex. Up to now only the singular perturbation problem for single laminates is well understood. To obtain lower bounds of the energy we believe that one should first study situations which lead to finite laminates. The experience gained there should give new insight for infinite laminates and hopefully lead to the derivation of lower bounds for the energy in this complex situation.

\section{Acknowledgements}

This research has been supported by the Deutsche Forschungsgemeinschaft (grant Wi 1251/11) and by the European Union (HCM Programme, contract ERBCHBICT 930744). I thank Professor J.M. Ball for his constant support and advice, Professor R.V. Kohn for bringing this problem to my attention and his encouragement and many hints during this work, Professor S. Müller for stimulating discussions at the beginning of this research, Professor M. Chipot for pointing out to me his work on the subject, and Professor S.D. Howison and 
the referees for many helpful comments. Finally, I acknowledge the hospitality of Courant Institute and the Institute for Advanced Study, Princeton.

\section{References}

[1] Aumann, R.J. And, Hart, S. 1986 Bi-Convexity and Bi-Martingales. Isr. J. Math. 54, 159-180.

[2] BALL, J.M. 1989 A version of the fundamental theorem for Young measures. In M. Rascle et al. (eds.), Lecture Notes in Physics 344, Springer, 207-215.

[3] BALl, J.M. And JAmes, R.D. 1987 Fine phase mixtures as minimizers of energy. Arch. Rat. Mech. Anal. 100, 13-52.

[4] BALl, J.M. And James, R.D. 1992 Proposed experimental tests of a theory of fine microstructure and the two-well problem. Phil. Trans. R. Soc. Lond. A 338, $389-450$.

[5] Bhattacharya, K. Firoozye, N.B. James, R.D. and Kohn, R.V. 1994 Restrictions on microstructure. Proc. Roy. Soc. Edinburgh Ser. A 124, 843-878.

[6] Cellina, A. And Perrotta, S. 1995 On a problem of potential wells. J. Convex Analysis 2, 113-125.

[7] Chipot, M. And Kinderlehrer, D. 1988 Equilibrium configurations of crystals. Arch. Rat. Mech. Anal. 103, 237-277.

[8] Chipot, M. And LÉcuyer, V. Analysis and Computations in the Four-Well Problem, preprint.

[9] Khachaturyan, A. 1983 Theory of Structural Transformations in Solids, Wiley.

[10] Khachaturyan, A. And Shatalov, G. 1969 Theory of macroscopic periodicity for a phase transition in the solid state. Soviet Phys. JETP 29, 557-561. 
[11] Kinderlehrer, D. And Pedregal, P. 1991 Characterizations of gradient Young measures. Arch. Rat. Mech. Anal. 115, 329-365.

[12] Kohn, R.V. And MÜLLER, S.1992 Branching of twins near an austenite-twinnedmartensite interface. Phil. Mag. A 66, 697-715.

[13] Kohn, R.V. And MüLleR, S. 1994 Surface energy and microstructure in coherent phase transitions. Comm. Pure Appl. Math. 47, 405-435.

[14] Kohn, R.V. And MülLeR, S. 1992 Relaxation and Regularization of Nonconvex Variational Problems. Rend. Sem. Mat. Fis. Univ. Milano 62, 89-113.

[15] Murat, F. 1981 L'injection du cône positif de $H^{-1}$ dans $W^{-1, q}$ est compacte pour tout $q<2$, J. Math. Pures Appl. 60, 309-322.

[16] Pedregal, P. 1993 Laminates and microstructure. Euro. J. Appl. Math. 4, 121149.

[17] Roitburd, A.L. 1978 Martensitic transformation as a typical phase transformation in solids. Solid State Physics 33, 317-390.

[18] Schreiber, C. 1994 Rapport de Stage D.E.A., Universität Freiburg.

[19] TARTAR, L. 1978 Compensated compactness and applications to partial differential equations. In R.Knops (ed.), Nonlinear Analysis and Mechanics, Pitman Research Notes in Mathematics, 39, 136-212.

[20] TARTAR, L. 1992 Some remarks on separately convex functions. In D.Kinderlehrer et.al. (eds.), Microstructure and Phase Transitions, Springer, 191-204.

[21] Winter, M. Lavrentiev phenomenon in microstructure theory, submitted. 